\title{
Evaluation of mechanisms responsible for glycaemic index values for cereal based food groups with a focus on slow release carbohydrates
}

\author{
Charlene Bailey and Klaus Englyst \\ Englyst Carbohydrates Ltd, Southampton, United Kingdom
}

\section{Abstract}

The glycaemic index (GI) concept has highlighted the detrimental metabolic impact of consuming foods high in rapidly released carbohydrates. However, diverse food properties such as slow release carbohydrates, viscous fibre, fructose, fat and protein can all result in foods with low GI values, with not all of them considered to be health beneficial.

The study aim was to identify the mechanisms responsible for the GI values for various food groups. Data was collated and evaluated for GI, presented here as mean (range), macronutrient composition and starch digestibility measures with a focus on slowly digestible starch expressed as percent available starch (\%SDS).

Wheat breads had high GI values for both white, 73 (59-88) and wholemeal, 70 (52-85). While wheat flour contains $>50 \%$ SDS, the moist heat conditions during baking gelatinises starch and most breads had low \%SDS 5 (3-10). Rye breads containing kernels had lower GI, 59 (41-78) and higher \%SDS, 16 (12-24). Cooked grains had diverse GI 57 (29-75), with a key determinant being kernel integrity, influenced both by grain type and cooking conditions. Typical GI and \%SDS values respectively for cooked grains were: white rice 65, 28; bulgur wheat 48, 40; pearl barley 29, 53. Pasta was similar in that matrix integrity determines starch digestibility and hence GI, 49 (40-57). Pasta thickness, ingredients and cooking time all influence \%SDS 37 (30-47). The fat and sugar in bakery products sometimes contributed to lower GI, 66 (55-92), while the \%SDS was low 5 (1-9). For biscuits, varied moisture content during baking impacts on the extent of starch gelatinisation explaining the range in \%SDS 24 (12-42) and GI 51 (47-56). Crackers are baked with high moisture and consequently had low \%SDS, 6 (5-7) and high GI, 67 (60-78). Breakfast cereals, produced by extrusion had low \%SDS, 3 (2-4), but sugar contents contributed to a wide GI range, 69 (46-87).

This study highlights the prevalence of rapidly digestible starch in many types of cereal based foods, due to processing techniques that result in easily dispersed food matrices and gelatinised starch. High fat and sugar products can have low or medium GI values despite having high contents of rapidly released carbohydrates, and as such GI could be considered an inconsistent measure of carbohydrate quality. Having information on starch digestibility profiles can identify the low GI foods that are rich in slow release carbohydrates, the consumption of which should be promoted.

\section{Conflict of Interest}

This study is self funded by Englyst Carbohydrates Ltd, a company providing analytical services to industry and academic institutions. 